\title{
PARTICLE BASED MODELLING OF MECHANICAL ALLOYING BY PARALLEL MONTE CARLO METHODS
}

\author{
Roland Reichardt, Fred Weinhold and Wolfgang Wiechert \\ Department of Simulation \\ University of Siegen \\ D-57068 Siegen, Germany \\ E-mail: roland.reichardt@uni-siegen.de
}

\section{KEYWORDS}

MPI, Parallelization, Cluster Computing, Mechanical Alloying, Monte Carlo

\begin{abstract}
Mechanical alloying (MA) is used to produce new materials which cannot be obtained by other production processes. Simplified two powders $A$ und $B$ are mechanically joined to a new powder $C$ which has new material properties. A particle based mathematical model for MA is developed for improving the mechanistic understanding of MA in a high energy ball mill. The model focuses on treatments from special milling devices and an investigation of breakage and fusion for ceramic materials. It was implemented by using parallel Monte Carlo methods for a large number of individual particles. To compare the simulation results with experimental data the system $\mathrm{SiC}$ and $\mathrm{Al}_{2} \mathrm{O}_{3}$ was investigated.

The aim of this paper is to improve the mechanistic understanding of MA in a high energy ball mill. Particularly the phenomena observed by milling of ceramic powders are investigated.
\end{abstract}

\section{SIMULATION CONCEPTS}

\section{Multi-Scale Approach}

The number of particles in a ball mill typically is in a dimension of $10^{12}$ and the number of milling balls is in the order of $10^{3}$. Clearly, to rigorously simulate the whole milling process, i.e. the powder particles together with the milling balls is impossible, because even to simulate hundreds of moving balls can take a very long time [16]. Therefore, a multi-scale approach is chosen in this work. In this approach the ball movements are simulated separately from the alloying behavior of the particles.

A high performance multi-body simulator of the ball mill which deals with the milling balls has been described in [3,19] (Figure 1). In this simulator the influence of the powder on the ball collisions is included by a phenomenological ball collision law. Based on this law the simulator can be used to compute the ball collision energy distribution in the milling process with a realistic number of balls. This distribution in turn is the input for the powder model described below.

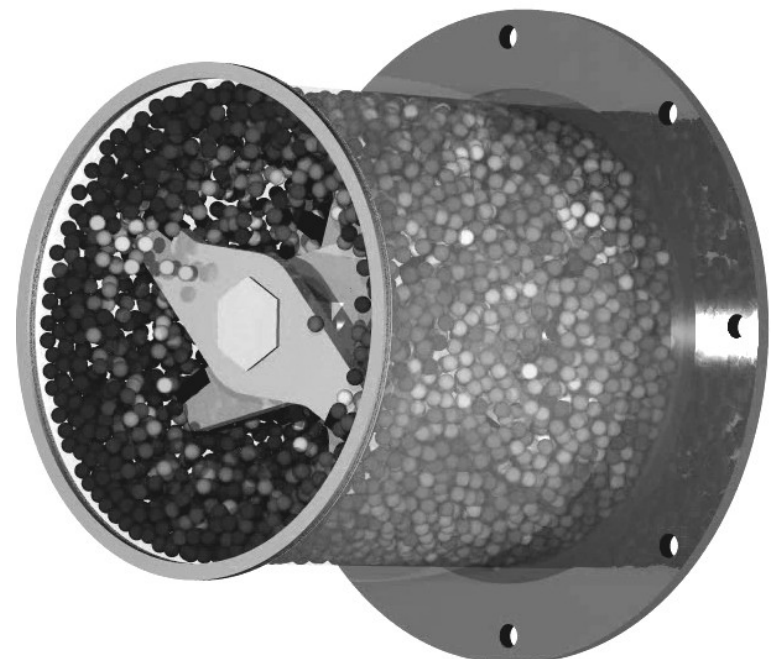

Figure 1: High energy ball mill simulation made by a high performance multi-body simulator [19]

\section{Distribution Versus Particle Based Simulation}

Particles characterized by the volume or mass distribution $U(x)$ where $x$ is the particle size are used in distribution based simulation $[7,18]$. The continuous time evolution of the distribution during the simulated MA is described by breakage and fusion functions. This leads to an integro-differential equation for $U(x)$ which must be solved numerically. To increase the number of different powder materials or to include further particle attributes like geometry or granularity parameters, this function has to be of a higher dimension, $U(x, y, z, .$.$) .$ Such a high dimensional function is impossible to handle directly in a numerical simulation.

To avoid these drawbacks, a new particle based method is proposed in this paper. This method simulates a representative population of single particles in a high energy ball mill and analyses it with statistical methods. Each particle is basically characterized by its particle size and chemical composition. However, these attributes can be easily increased in number so that nearly every number and type of attributes can be handled.

A Monte Carlo method is chosen to represent the breakage and fusion events stochastically. Herein the fracturing and fusion probabilities which are specified in the distribution based simulation are interpreted as fracturing and fusion probabilities of single particles. 


\section{Basic Algorithm}

The simulation algorithm starts with the creation of particles which will be fractured and fused during the simulated MA process. Initially, these particles are stored in a list along with the associated attributes (Figure 2). All parts of the algorithm then take place in an iterative loop, until a termination condition is reached. For example, the simulation can end if a given stopping time is reached or the resulting distributions reach a stationary state.

Each step in the loop can be interpreted as a time period. The length of this period depends on the number of simulated particles, because more particles in a mill imply that more particles will be involved in the milling process per unit of time.

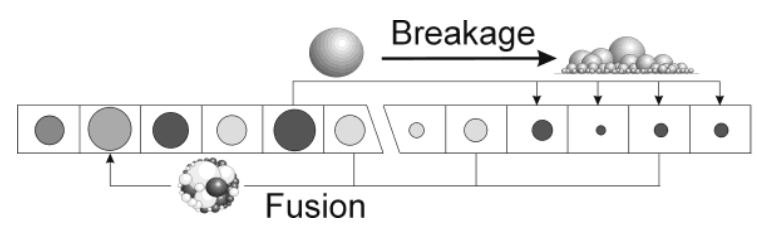

Figure 2: Particle list: fragments of a particle will be added to the particle list, whereas fusion particles will be removed from the list and their mass totalized to a single particle.

For breakage, one of the particles has to be chosen randomly. Depending on the particle parameters, a breakage probability will be calculated. This function is called the selection function. A random number is generated to decide if the chosen particle really breaks. If so, the breakage function - again by using random numbers - determines which fragments will be produced. Those fragments are new particles which have to be included in the simulation like other particles.

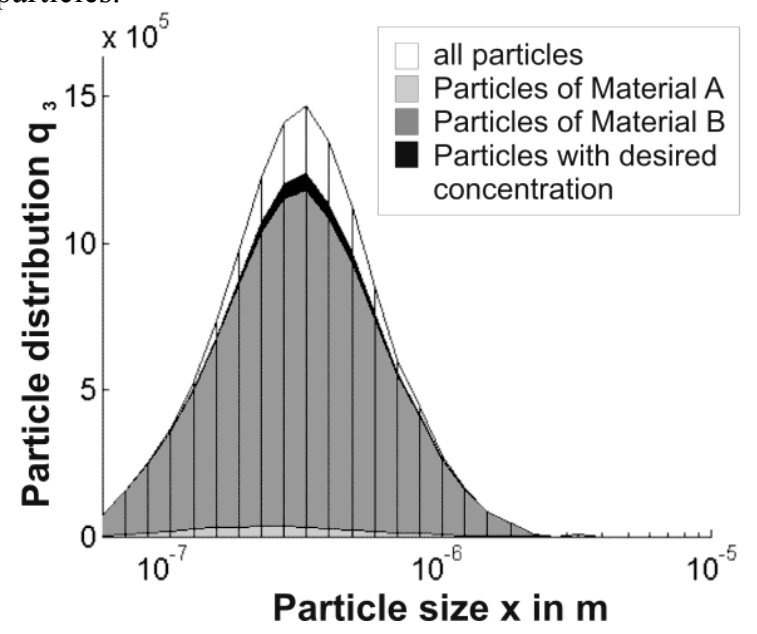

Figure 3: Distribution of simulated particles classified by material composition. White: all particles; Light grey: Particles close to the composition of raw Material A; Dark grey: Particles close to the composition of raw Material B; Black: Particles close to desired alloy composition.
For fusion, several particles have to be chosen. Now, the decision for or against fusion will be made by a welding function which will be compared with a random number. The chosen particles will be removed from the list and their volumes and compositions will be attributed to a single particle.

To update the powder statistics, each particle will be added to a histogram, which sorts the particles by size and their material components. In this diagram the particles can also be classified as particles of (almost) the composition of the raw materials or particles which are close to the desired alloy composition. A result is shown in Figure 3

\section{MODELLING CONCEPTS}

\section{Phenomenological versus Physical Modeling}

Phenomenological functions which are difficult to interpret are often defined to model the breakage and fusion processes in a high energy ball mill [10]. One reason for this black box approach is that it is surprisingly difficult to establish correlations between milling treatments in different devices [12]. The mathematical model developed in the following can use more physical information represented by the ball collision energy distribution taken from the simulated mill. This distribution is transformed to a particle stress distribution for the powder particles between the colliding balls. Only the relation between powder particle stress and breakage probability is modeled in a phenomenological way. Nevertheless - like in other established models - some common assumptions and abstractions are still required:

- each contact will be handled like a ball-powder-ball contact, i.e. ball-wall contacts are not distinguished;

- all particles which are involved in the alloying process are on the surface of the balls or the mill;

- all particles are spherical, i.e. their size already determines their shape;

- the material concentration in a particle is homogeneous, i.e. the detailed morphology of single particles is not considered;

- the pressure in the powder between the ball surfaces is in the order of magnitude of the pressure on the surface.

\section{Stress Probability Distribution}

The stress probability distribution for colliding balls characterizes the treatment of the particles in a mill. The development of the stress probability for a specific mill is based on the relative ball to ball impact velocity [3] (Figure 4) for specific milling conditions and the classical Hertz theory of impact. It will be determined which pressures in a particle will be reached during the grinding process. 

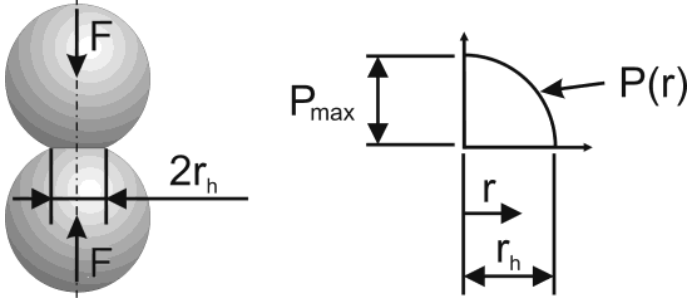

Figure 4: Stress between the grinding balls determined by the Hertz Pressure equations [19]

The first step is to approximate the average force, F, between the contact bodies from the relative impact velocity $\Delta v,[3]$ with

$$
\mathrm{F}=\mathrm{m}_{\mathrm{b}} \frac{\Delta \mathrm{v}}{\tau}
$$

where $m_{b}$ is the mass of the balls and $\tau$ the contact time. This time period $\tau$ can be calculated with [19]

$$
\tau=2.94\left(\frac{15}{8} \frac{\mathrm{m}_{\mathrm{b}}}{\sqrt{\mathrm{R}_{\text {eff }}} \mathrm{E}_{\text {eff }}}\right)^{\frac{2}{5}}\left(\frac{\Delta \mathrm{v}}{2}\right)^{-\frac{1}{5}}
$$

where $R_{\text {eff }}$ and $E_{\text {eff }}$ for a centric impact are defined as

$$
\mathrm{R}_{\text {eff }}=\frac{1}{2} \mathrm{R}_{\mathrm{b}}
$$

and

$$
\mathrm{E}_{\text {eff }}=\frac{1}{2} \frac{\mathrm{E}}{1-\mathrm{v}^{2}}
$$

$R_{b}$ is the radius of the balls in the mill, $E$ is Young's modulus and $v$ is Poisson's number. In the next step, the contact radius (see Figure 4$), r_{h}$, and the maximum pressure, $P_{\max }$, between the surfaces can be calculated by

$$
\mathrm{P}_{\max }=\frac{2}{3} \frac{\mathrm{F}}{\pi \mathrm{r}_{\mathrm{h}}^{2}}
$$

Described by Hertz's theory, only the centre of the contact area has the maximum pressure, which drops to zero at the border of the contact area. The pressure progression for a circular contact area can be given by

$$
\mathrm{P}=\mathrm{P}_{\max } \sqrt{1-\left(\frac{\mathrm{r}}{\mathrm{r}_{\mathrm{h}}}\right)}
$$

where $r$ is the distance from the pressure centre (Figure 4)

A linear increase of the contact radius increases the area quadratically. Hence, particles are less often in the impact centre than in the border area where the pressure is lower. Thus even a high energy impact imposes only a small pressure on the peripherical particles in the contact area. This behavior again, is modeled stochastically. The resulting stress probability distribution $R$ is shown in Figure 5

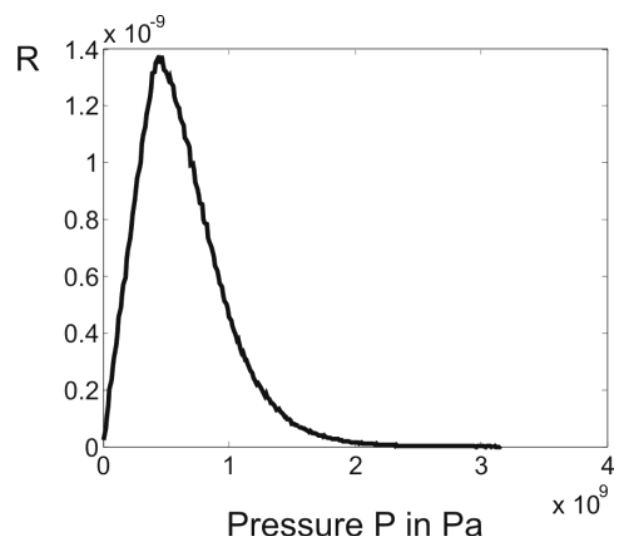

Figure 5: The stress probability distribution $R$ calculated by the Hertz Pressure equations [19] and the simulated relative impact velocity used in this investigation [3].

\section{Selection Function}

The selection function, $S(d)$, determines the amount of particles which break in a period of time. This function has been introduced to describe the efficiency of grinding mills [10]. In this paragraph, the selection function will be built by the combination of the stress probability distribution and the Griffith-equation [11]

$$
\sigma_{c}=Y \frac{K_{I c}}{\sqrt{\pi a}}
$$

Which determines a breakage stress, $\sigma_{c}$, for a particle by using mainly the length of the worst crack, $a$. Also a geometrical factor, $Y$, and a material constant , $K_{I C}$, is required. It will be assumed that the length of the worst crack in a particle is proportional to the size of the particle. This leads to the Hall-Petch relationship $[2 ; 7]$

$$
\sigma_{\mathrm{y}}(\mathrm{d})=\mathrm{k}_{\mathrm{m}}+\mathrm{k}_{0} \mathrm{~d}^{-1 / 2}
$$

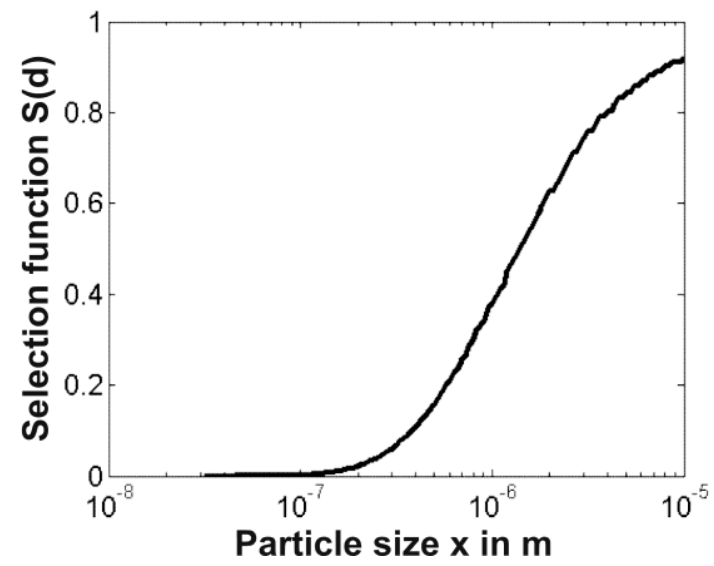

Figure 6: Selection function depending on particle size: Particles smaller than $107 \mathrm{~m}$ will not break, whereas particles bigger than 105 will be nearly always broken.

In this relationship, $k_{m}$ and $k_{0}$ are material and size constants. The yield strength $\sigma_{y}$ is directly connected to the particle diameter $d$. With the stress probability distribution $R$ (see Figure 5), it is now possible to 
calculate the probability that a special particle will break, because if the stress is higher than the yield strength $\sigma_{y}$ then it will break. This can be described by

$$
\mathrm{S}(\mathrm{d})=\int_{\sigma_{\mathrm{y}}(\mathrm{d})}^{\infty} \mathrm{R}(\sigma) \mathrm{d} \sigma
$$

The result for each relevant particle size is shown in Figure 6. As expected, small particles do not break, but almost all big particles will break.

\section{Breakage Function}

The breakage function is the rate at which particles are selected by the selection function of the ground component become particles of another size. This section will be based on observations, because the theoretical understanding of breakage behaviors is still under examination [20].

Some investigations were done about breaking of glass balls [21] in a size range from $38 \mu \mathrm{m}$ to $1000 \mu \mathrm{m}$. One result of this investigation was that independent of the particle size the volume distribution can be linearly approximated in a wide range. With this in mind the following breakage function $B$ was build:

$$
\mathrm{B}\left(\mathrm{d}_{\text {min }}, \mathrm{x}\right)=\left(\mathrm{k}_{\mathrm{e}}^{2}-3 \mathrm{k}_{\mathrm{e}}+2\right) \mathrm{d}_{\text {min }}^{\mathrm{k}_{\mathrm{e}}-2} \frac{\mathrm{x}-\mathrm{d}_{\text {min }}}{\mathrm{d}_{\text {min }}}
$$

for $x>d_{\min }$ and $k_{e}>2$, where $d_{\min }$ is the minimal particle size, $k_{e}$ a constant and $x$ the considered particle size. However, it should be kept in mind that other types of experimentally determined functions can be substituted for $B$.

\section{Fusion Function}

Like for breakage, the process of fusion, especially the fusion of ceramic particles, is not yet well understood. However, the physically motivated particle stress distribution can again be separated from a phenomenological model for the relation between stress and fusion probability. Depending on the stress probability distribution $R$, a critical stress $\sigma_{w}$ can be defined, which has to be reached until particles agglutinate together. The fusion will be represented with the threshold constant $W$ given by

$$
\mathrm{W}=1-\int_{0}^{\sigma_{\mathrm{W}}} \mathrm{R}(\sigma) \mathrm{d} \sigma
$$

The fusion constant $W$ is comparable with the critical stress value $\sigma_{w}$. A value of zero for the fusion constant $W$ correlates to infinite critical stress and alloying will not occur.

In the contact area thousands of particles are pressed together. Therefore in this simulation, $n_{W}$ particles will build a new one. This simple model also includes that smaller particles fuse together more often than big particles, because they are more numerous.

\section{RESULTS}

\section{Simulation parameters}

By using the Monte Carlo simulation, the accuracy of the results often depends on the number of simulated objects i.e. the number of simulated particles. To estimate the repeat accuracy, about $10^{4}$ particles are simulated twice with identical start conditions. The results didn't show significant differences. Therefore, it seemed as if this particle number is high enough. In the simulation, good results could be reached with $10^{6}$ to $10^{7}$ particles.

The simulation was carried out on 12 computers in parallel to minimize the simulation time. Therefore, the simulation time was only in the range of 5 to 10 minutes for 60 minutes of simulated milling of $10^{7}$ particles.

\section{Model Verification}

The first simulations are used to verify the model and their implementation. Figure 7 shows simulation results for a fictive starting particle population which should clarify the milling behaviors of breaking and fusing. For a more thorough verification of the simulation all parameters have been varied. For the sake of brevity this will be limited here to two parameters of interest. The critical stress constant $W$ for fusion and the number of particles $n_{W}$ which will be alloyed together are considered. For those parameters, the value of the parameter, the average particle size and the associated standard deviation is compared. The average particle size in the steady state and the associated standard deviation are presented in the same plot, like in Figure 7 , where the centre line represents the average particle size and the distance to the external lines indicates the standard derivation.

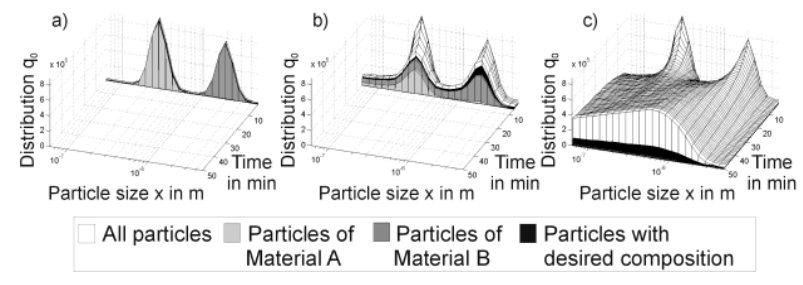

Figure 7: Illustrative example simulation with fictive starting distribution to visualise the simulation behaviour: a) Starting distribution of two different materials with different average particle sizes; b) reducing of the average particle size of each material by fusion and breakage; c) In this simulation after milling of 50 simulated minutes resulted in only a low value of desired particles. Another starting material weight ratio has to be chosen

The critical stress constant $W$ is varied between 0 and 1 . The results are plotted against the number of resulting particles in the stationary state (Figure 7a) and against the size of these particles (Figure 8b). Clearly, the number of particles decreases because larger aggregates are formed for high fusion rates. Simultaneously, the mean particle size increases almost linearly while the distribution becomes broader. 

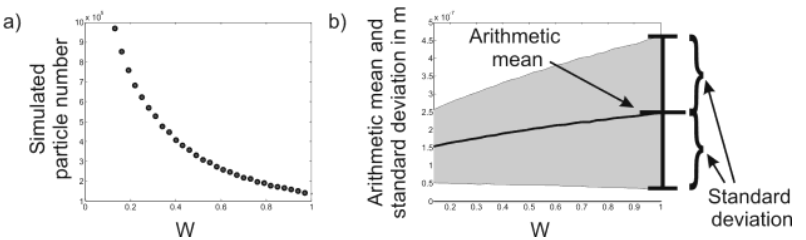

Figure 8: Effect on the steady state of increasing of the critical stress constant, $W:$ a) Number of particles remaining in steady state; b) Corresponding mean particle size and standard derivation.

Likewise, the number of particles $n_{W}$ which will be alloyed together is varied between 2 and 20. The steady state results are plotted against the number of particles (Figure 9a) and against the particle size (Figure 9b). It turns out that $n_{W}$ has a qualitatively similar effect as $W$. For a critical stress constant $W$ of 0 , fusions of particles do not occur. Because the particles would only break the average particle size is only limited by the minimal particle size $d_{\min }$. The other extreme is the permanent fusion of many particles. This results in an interruption of the simulation, because all particles are joined together to one particle.
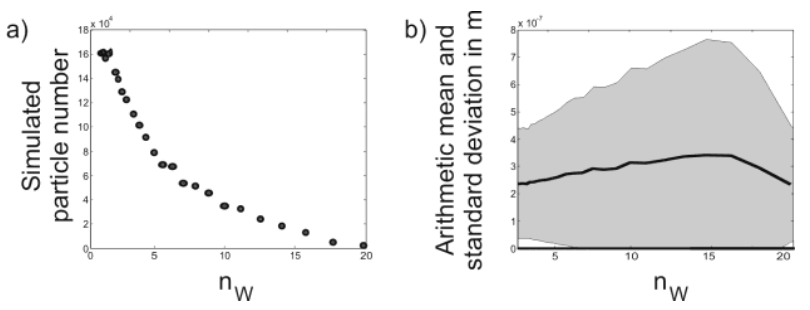

Figure 9: Results by increasing of the number of particles $n_{W}$ which will be alloyed together: a)

Decreasing of the number of particles to zero; b) A linear increasing of the mean particle size and the standard derivation until abort because of too little particles.

\section{Simulation Results Compared to Measured Data}

To verify the simulation results, they are compared to the measured data in Figure 10 [9]. The measured particle size distributions in a high energy ball mill are recorded for a grinding time of $0,15,30$ and 60 minutes. After an initial increase of the average particle size, a steady state was reached. After 30 minutes, the milled powders had reached the required distribution and consistence and after 60 minutes no differences to that state were detectable.

For the simulation, the model parameters are chosen to minimize the differences between simulation and recorded data. The best fit to the data is achieved for a critical stress threshold $W=1$ and a number of alloyed particles $n_{W}=2$.
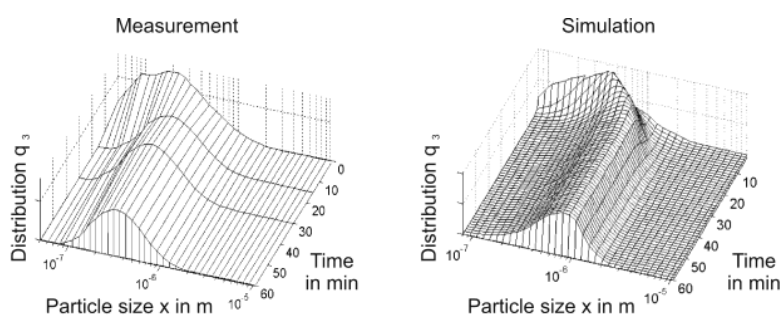

Figure 10: Comparison of measured data with the simulation results

\section{DISCUSSION}

\section{Particle based simulation}

The particle based simulation approach proved to be well suited for the MA process. The major advantage over distribution based methods is the flexibility to describe each particle by a high dimensional set of attributes. In this contribution only the size and composition have been used but other parameters like shape parameters (e.g. assuming ellipsoid shapes of the particles) or parameters describing the average size of crystallites in the particle are possible. By this means a description of particles up to a certain detail can be achieved.

Another new idea in the simulation of MA is the multiscale approach which separates the mechanical multi body system of the milling balls from the powder process. Both processes are loosely coupled via the collision energy distribution (downwards) and the ballto-ball collision energy dissipation (upwards). Coupling both approaches the complete process can be modeled. In this investigation only the downward coupling was implemented by assuming the energy dissipation law. However, from experiences with a variation of the dissipation law it is known that the 'upward' influence will not be dramatic.

The simulation effort for the particle approach turned out to be surprisingly low so that even on a single processor simulation runs can be performed with reasonable computing time. Moreover, the performance can be significantly increased by parallelization on a compute cluster. One problem of the particle approach is that at the tails of the particle distribution only a few particles a found as representatives. Consequently, the statistics for these very large and very small particles has a large uncertainty. However, as long as the focus of interest lies on the bulk of the particles (as in this investigation) this well known effect has little influence.

\section{Modeling assumptions}

In this work the share of first principles in the modeling of the MA process could be significantly increased due to the multi-scale approach. However, the assumed laws that govern the breakage and fusion events are still of a phenomenological nature. The benefit of the physically motivated multi-scale approach is that the influence of phenomenological assumptions on the model prediction 
could be reduced because the ball collision energy distribution already determines the breakage and fusion distributions to a certain extent.

Clearly, different mathematical types of phenomenological breakage and fusion laws are still possible. However, some trial experiments showed that the precise shape of the phenomenological functions had only minor influence on the qualitative results as long as the principal characteristics (like monotonicity) is similar. The obtained fusion probability of $W=1$ must also be interpreted in this direction. Clearly, it cannot really be the case that two particles selected for fusion will always do it. However, even a ten times lower probability would support the hypothesis of low fusion energy. Moreover, the value of $W$ cannot be precisely estimated by a stochastic particle method and a change in the phenomenological laws might also lower this value.

\section{REFERENCES}

[1] N. Stubičar, A. Tonejc, M. Stubičar, Microstructural evolution of some $\mathrm{MgO}-\mathrm{TiO} 2$ and $\mathrm{MgO}-\mathrm{Al} 2 \mathrm{O} 3$ powder mixtures during high-energy ball milling and post-annealing studied by X-ray diffraction. Journal of Alloys and Components 370 (2004) 296-301

[2] C.C. Koch, Intermetallic matrix composites prepared by mechanical alloying - a review. Materials Science and Engineering A 244 (1998) 39-49

[3] R. Reichardt, W. Wiechert, Simulating High Energy Mills using Discrete Events, Simulation News Europe, Issue 38/39 (2003) 3-9

[4] L.B. Kong, J. Ma, T.S. Zhang, W. Zhu, O.K. Tan, $\mathrm{Pb}$ (ZrxTi1-x)O3 ceramics via reactive sintering of partially reacted mixture produced by a highenergy ball milling process. Journal Material Research, Vol. 16, No. 6 (2001) 1636-1643

[5] Chung-Kwei Lin, Shi-Shen Hong, Pee-Yew Lee, Formation of NiAl-Al2O3 intermetallic-matrix composite powders by mechanical alloying technique. Intermetallics 8 (2000) 1043-1048

[6] H. Wang, L.Z. Ouyang, M.Q. Zeng, M. Zhu, Direct synthesis of $\mathrm{MgCNi3}$ by mechanical alloying. Scripta Materialia 50 (2004) 1471-1474

[7] P.R. Soni, Mechanical Alloying. Fundamentals and Applications. Cambridge International Science Publishing (2000)

[8] P. Ju. Butyagin, The chemocal forces in mechanical alloying. Materials Science Forum Vol. 88-90 (1992) 695-702

[9] E. Kern, $\mathrm{PhD}$ thesis, Herstellung und Charakterisierung von thermisch gespritzten A12O3-SiC-Nanokomositschichten. University of Siegen, Germany, 2004

[10] N. Kotake, K. Suzuki, S. Asahi, Y. Kanda, Experimental study on the grinding rate constant of solid materials in a ball mill. Powder Technology 122 (2002) 101-108
[11] Iona D, Marinescu, Hans K. Tonshoff, Ichiro Inasaki, Handbook of ceramic grinding and polishing. Noyes publications, Park Ridge, New Jersey, U.S.A. (2000)

[12] F. Delogu, M. Monagheddu, G. Mulas, L. Schiffini, G. Cocco, Impact characteristics and mechanical alloying processes by ball milling: experimental evaluation and modelling outcomes. International Journal of Non-Equilibrium Processing, Vol. 11 (2000) 235-269

[13] Yong Yang, Jie Lan, Xiaochun Li, Study on bulk aluminum matrix nano-composite fabricated by ultrasonic dispersion fo nano-sized $\mathrm{SiC}$ particles in molten aluminium alloy. Materials Science and Engineering A 380 (2004) 378-383

[14] Guoping Ling and Junhui He: The influence of nano $\mathrm{Al} 2 \mathrm{O} 3$ additive on the adhesion between enamel and steel substate. Materials Science and Engineering A 379 (2004) 432-436

[15] Stefan Hüfner, Photoelectron Spectroscopy Principles and Applications. Springer-Verlag, Berlin Heidelberg New York (2003)

[16] W. Wiechert, H. Mournier, D. Hoppe, Modeling the Mechanical Alloying Process I. Troch, F. Breitenecker (Eds.), 2nd IMACS Symposium on Mathematical Modelling, Vienna, February 2-4, (2000) 685-690

[17] Thorsten Pöschel, Granular gas dynamics. Springer-Verlag, Berlin (2003)

[18] Winfried Schröder, MM Maschinenmarkt - Das Industrie Magazin. Rechenmeister. Vogel Industrie Medien, Würzburg 34 (2002) 23

[19] Reichardt, R.: Ereignisdiskrete Simulation einer Hochenergie-Kugelmühle, Dissertation, 2005, urn:nbn:de:hbz:467-1914

\section{AUTHOR BIOGRAPHIES}

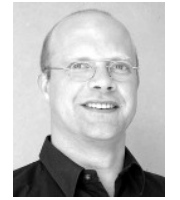

ROLAND REICHARDT studied mechanical engineering at the University of Siegen, Germany. He worked from 1997 to 2001 for a mechanical process engineering company of ball mills in the company management. In 2002, he returned to the University of Siegen and obtained his doctoral degree in 2005. Since 2006, he is additionally lecturer at the Niederrhein University of Applied Sciences.

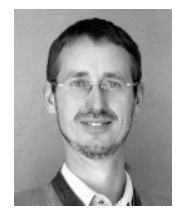

WOLFGANG WIECHERT studied mathematics and computer science at the University of Bonn and obtained his diploma degree in 1985. Subsequently, he joined the Department of Theoretical Biology at the University of Bonn, where he obtained his $\mathrm{PhD}$ in 1991. From 1991 to 1996 , he worked in the Institute of Biotechnology at the Jülich Research Center, where he earned his postdoctoral lecturer's qualification. Since 1996, he has been professor for simulation at the Institute of Systems Engineering at the University of Siegen 